\title{
ANNEALING EFFECT OF EQUIATOMIC CoPt ALLOY FILMS
}

\author{
R. GonTARZ, T. LuCińSKI \\ Institute of Molecular Physics, Polish Academy of Sciences \\ Smoluchowskiego 17, 60-179 Poznań, Poland \\ L. UBA, S. UBA \\ Institute of Physics, Warsaw University Branch in Białystok \\ Lipowa 41, 15-424 Białystok, Poland \\ AND YU.V. KuDRYAVTSEV
}

Institute of Metal Physics Ukr. AS, 36 Vernadsky st., 252142 Kiev, Ukraine

Polycrystalline as-sputtered CoPt alloy films near the equiatomic composition were characterized as consisting of a disordered fcc phase with preferred texture along [111] orientation perpendicular to the film plane. The film annealing at $600^{\circ} \mathrm{C} / 16 \mathrm{~h}$ induces the disorder-order phase transition confirmed by X-ray diffraction. As a result of the transition an appearance of the ordered tetragonal (fct) phase of $\mathrm{AuCuI}$ type with $c / a=0.974$ is observed. The $c$-axis is the easy axis of magnetization. Both longitudinal and polar Kerr hysteresis loops reveal a dramatic increase in the coercivity from 0.1 to $4.5 \mathrm{kOe}$ in annealed $\mathrm{CoPt}$ alloy films due to ordering.

PACS numbers: 81.40.Ef, 75.70.Fr, 61.50.Ks

\section{Introduction}

CoPt alloys form a complex solid solution [1]. The as-quenched alloys reveal disordered face centered cubic ( $\mathrm{fcc}$ ) structure but on annealing at several hundred degrees Celsius, and depending upon composition, three distinct allotropic modifications appear. At about 25 at.\% Pt, there is a transformation from fcc to a hexagonal close packed (hcp) structure; both phases are disordered. Alloys in the equiatomic range are well known as permanent magnetic materials of very high energy product. These extensively studied $[2,3]$ alloys can be produced with coercivities of several thousand oersteds depending on processing conditions. By prolonged annealing at 600 to $700^{\circ} \mathrm{C}$, the disordered fcc phase transforms to an ordered face centered tetragonal (fct) structure of AuCuI type. The coercivity is small for disordered fcc phase of this composition, and increases with annealing 
when about one tialf of the fcc phase is transformed to the ordered fct phase, and then decreases to a small value when the transformation to fct is complete. For compositions of about 75 at.\% $\mathrm{Pt}$, the disordered fcc is transformed to an ordered fcc phase. In recent years $\mathrm{CoPt}$ thin films attained great interest. Their high corrosion resistance combined with the optimized magnetic properties makes them good candidates for magnetic recording applications. However, they have some drawbacks. One of them is the relatively small coercivity. Enhancement of the coercivity to $4.5 \mathrm{kOe}$ at $T_{\mathrm{s}}=300^{\circ} \mathrm{C}$ [5] has been attained by deposition of the $\mathrm{Co} / \mathrm{Pt}$ multilayers onto substrate heated up to $500^{\circ} \mathrm{C}$.

The aim of this paper is the careful examination of the structural transformation from fcc disordered to fct ordered phase of equiatomic CoPt alloy film with particular attention to the correlation between crystalline structure and magnetic properties.

\section{Experimental}

CoPt alloy films have been deposited by DC cosputtering using a face-to-face system previously described [4]. The glow discharge was magnetic-field stabilized. The argon pressure during deposition was about $60 \mathrm{mPa}$; the initial vacuum was better than $0.2 \mathrm{mPa}$. The films were deposited onto water-cooled glass substrates. The deposition rate was about $0.5 \mathrm{~A} / \mathrm{s}$. The composition of the deposited films was adjusted by varying the cathodes potentials. In this way, we were able to obtain alloy films of equiatomic compositions. The chemical compositions of these films were determined with X-ray fluorescence analysis (XRF). For XRF measurements the samples were excited by tungsten characteristic radiation. The EDX system was applied in the X-ray detection. The compositions were determined from the characteristic fluorescence of Co $K_{\alpha}$ and $\mathrm{Pt} L_{\alpha}$ radiation. The diffractograms were taken with standard X-ray diffractometer using the $\vartheta-2 \vartheta$ mode with Co $K_{\alpha}$ radiation. The phase transformation $\mathrm{fcc} \Rightarrow \mathrm{fct}$ was determined with X-ray diffraction from (200) and (002) and/or (311) and (113) reflections.

Magnetooptical polar and longitudinal Kerr hysteresis loops were measured up to $870 \mathrm{~K}$ in saturating external magnetic field up to $18 \mathrm{kOe}$ in fully automated Kerr spectrometer with the use of the $\lambda=0.58 \mu \mathrm{m}$ laser radiation.

\section{Results and discussion}

Figure 1 shows an $\mathrm{X}$-ray diffraction of the $\mathrm{Co}_{51} \mathrm{Pt}_{49}$ alloy film before and after annealing using a Co $K_{\alpha}$ radiation. The as-grown sample shows high-angle diffraction peaks of the fcc structure: (111), (200), (311) and (222), but only the peak (111) has a strong preferential orientation. No peaks which reflect the ordering in the sample were observed in X-ray diffraction before annealing. After annealing in vacuum of $0.2 \mathrm{mPa}$ at a temperature of $550 \mathrm{~K}$ all the peaks of the fcc structure were shifted to higher angles $2 \vartheta$ and the half-width of the X-ray peaks was reduced by a factor of two. The basic [100] crystallographic axes are supposed to be randomly distributed and forming an angle $\gamma=35.26^{\circ}$ with the thin film surface (inset in Fig. 1). Further annealing at $870 \mathrm{~K}$ during 16 hours induced a 


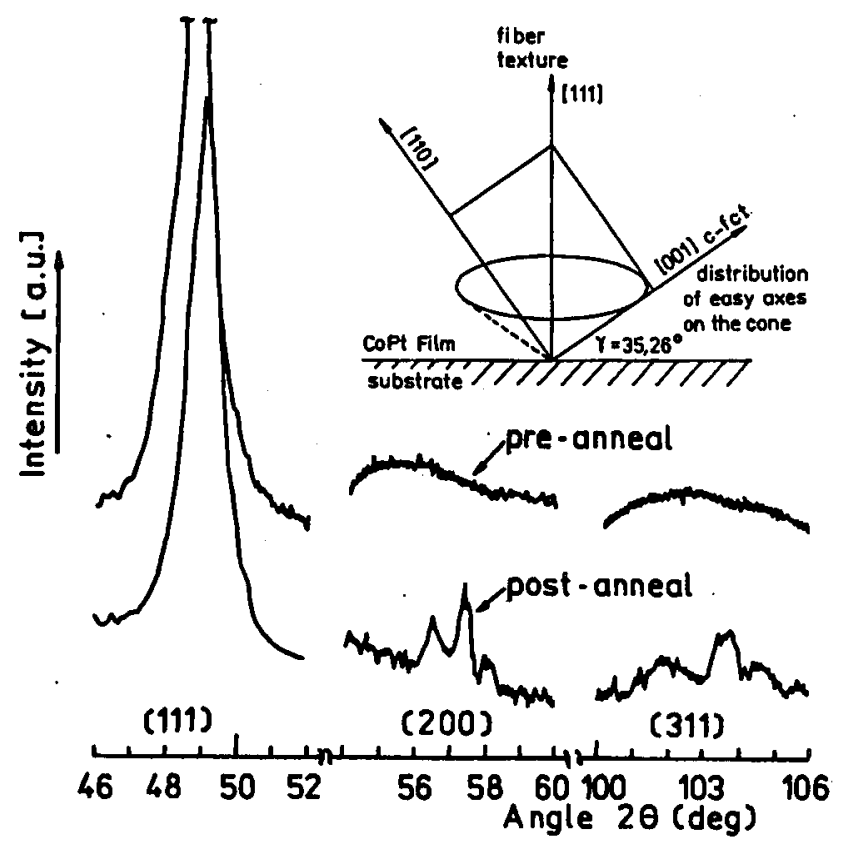

Fig. 1. X-ray diffraction of the $\mathrm{Co}_{51} \mathrm{Pt}_{49}$ alloy film before and after annealing using Co $K_{\alpha}$ radiation. The inset depicts the distribution of the easy axis directions (\| the $c$-axis of fct structure) in strongly textured polycrystalline $\mathrm{CoPt}$ films in the ordered state fct $(0)$.

transformation from a disordered $f c c(d)$ phase to an ordered fct(o) phase. Effect of this heat treatment on the lattice constants of $\mathrm{Co}_{51} \mathrm{Pt}_{49}$ alloy film is shown in Table. After transformation one of the [100] axes fcc structure becomes the $c$-axis of the fct structure, which at the same time is the easy axis of the uniaxial magnetocrystalline anisotropy of the CoPt alloy. Hence, we can arrive at a conclusion that the effective anisotropy direction in the film with a [111] texture and with the fct(o) structure lies on the conicid. Thus, the film magnetization process in and out of the film plane will occur by rotation of the magnetization vector $M_{\mathbf{s}}$. The reversible magnetization process will be dependent on the $f c c(d)$ phase, which has remained after the transformation. It is known [3] that in bulk materials the fcc(d) phase allows one to reach the maximum of the coercivity for about $50 \%$ structural transformation.

The structural changes that occurred upon annealing were accompanied by changes in magnetic properties. Figure $2 \mathrm{a}$ and $2 \mathrm{~b}$ illustrates the polar and longitudinal Kerr loops before and after annealing. As can be seen in the figure, before annealing the sample was much easier magnetized in the plane of the film than in the direction perpendicular to the plane. Almost no hysteresis loop was observed in and out of the film plane; the coercivity $H_{c}^{\perp}=120 \mathrm{Oe}$ and $H_{c}^{\|}=130 \mathrm{Oe}$, respectively. The hysteresis loop measured after the anneal shows a dramatic change 
TABLE

Effect of heat treatment at a temperature of $870 \mathrm{~K}$ on the lattice constants of $\mathrm{Co}_{51} \mathrm{Pt}_{49}$ alloy film, thickness $2500 \AA$; $a, c, d$ - lattice constants in $\AA$.

\begin{tabular}{l|l|l|l}
\hline \hline Film $\mathrm{Co}_{51} \mathrm{Pt}_{49}$ & \multicolumn{1}{|c|}{$(111)$} & \multicolumn{1}{c|}{$(200)$} & \multicolumn{1}{c}{$(311)$} \\
\hline pre anneal & $d_{111}=2.192$ & $d_{200}=1.908$ & $d_{311}=1.148$ \\
& $a=3.796$ & $a=3.816$ & $a=3.809$ \\
& & fcc & \\
post anneal & $d_{111}=2.155$ & $d_{200}=1.862$ & $d_{311}=1.137$ \\
$870 \mathrm{~K}-16 \mathrm{~h}$ & $a=3.732$ & $a=3.725$ & \\
& & $\mathrm{fct}$ & \\
& & $d_{002}=1.839$ & $d_{113}=1.115$ \\
& & $c=3.678$ & \\
& & $d_{200}=1.889$ & $d_{311}=1.123$ \\
& & $a=3.779$ & \\
& & $c / a=0.973$ & $c / a=0.972$
\end{tabular}
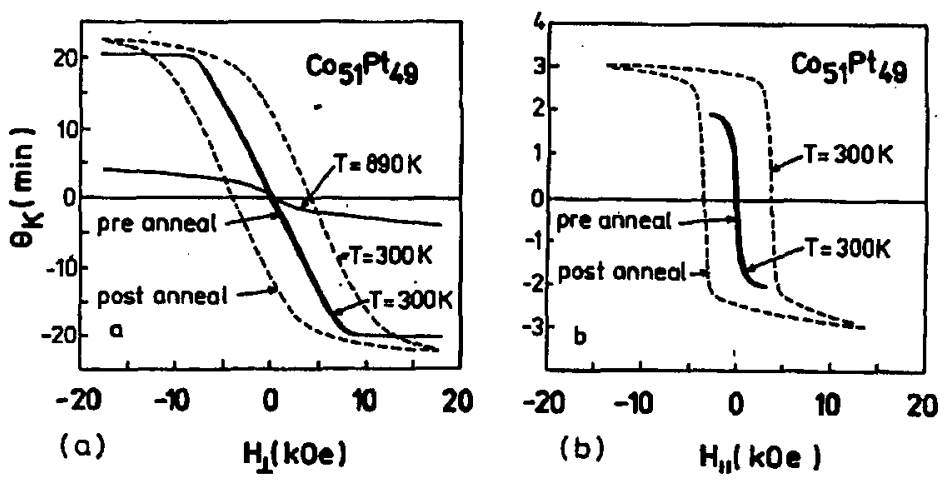

Fig. 2. Magnetooptical polar (a) and longitudinal (b) Kerr hysteresis loops taken up to $890 \mathrm{~K}$ for $\mathrm{Co}_{51} \mathrm{Pt}_{49}$ alloy film before and after annealing.

in the Kerr hysteresis loops. The films become much more easily magnetized out of the plane of the sample, and more difficult to magnetize in the plane. In addition, we observed hysteresis loops with a coercive field of $4.1 \mathrm{kOe}$ out and $3.6 \mathrm{kOe}$ in the film plane. As can be seen in Fig. 2, the $18 \mathrm{kOe}$ magnetic field is insufficient to saturate the magnetic moment in such a direction. It is well known that the microstructure and magnetic properties of the ordered CoPt intermetallic phase are sensitive to annealing time and temperatures. Magnetic torque measurements were performed on the annealed sample for which the hysteresis loop is shown in Fig. 2. For all magnetic fields applied, up to $18 \mathrm{kOe}$, rotational hysteresis curves 
were obtained, i.e., it was not possible to completely saturate the sample. This indicates that the sample has a magnetic net of the easy axis perpendicular to the sample surface.

\section{Conclusion}

The magnetic properties of films have been correlated with the allotropic modifications of the CoPt alloy system. The properties of the equiatomic $\mathrm{CoPt}$ were found to be similar to those of bulk alloy: their coercivity increases to high values upon heat treatment, as an ordered tetragonal phase separates from the cubic phase.

The main results of our paper characterizing the annealed $\mathrm{CoPt}$ films can be summarized as follows:

(i) for the first time a structural transformation $\mathrm{fcc}(\mathrm{d}) \Rightarrow \mathrm{fct}(\mathrm{o})$ upon annealing at $870 \mathrm{~K}-16 \mathrm{~h}$, was observed in thin CoPt alloy film.

(ii) we found the high magnetic anisotropy with the easy axis off the film plane,

(iii) both longitudinal and polar hysteresis loops revealed high coercivities of 3.6 and $4.1 \mathrm{kOe}$, respectively.

It is worth stressing that the coercive field enhancement observed in $\mathrm{CoPt}$ annealed films can be very useful for the magnetic recording media.

\section{Acknowledgment}

One of us (L.U.) wishes to thank for financial support by the Committee for Scientific Research, grant No. 2291/2/91.

\section{References}

[1] A.S. Darling, Platinum Met. Rev. 7, 96 (1963).

[2] D.J. Craik, Platinum Met. Rev. 16, 129 (1972).

[3] Ya.S. Shur, L.G. Magat, G.V. Ivanova, A.I. Mitsek, A.S. Ermolenko, O.A. Ivanov, Fiz. Met. Metalloved. 26, 241 (1968).

[4] R. Gontarz, T. Luciński, J. Magn. Magn. Mater. 101, 253 (1991).

[5] S. Shiomi, T. Nakabayashi, M. Okada, T. Kobayashi, M. Masuda, Jpn. J. Appl. Phys. 32, 791 (1993). 